\title{
Efficacy study of olmesartan medoxomil on coronary atherosclerosis progression and epicardial adipose tissue volume reduction in patients with coronary atherosclerosis detected by coronary computed tomography angiography: study protocol for a randomized controlled trial
}

Ying Zhou, Feng Tian, Jing Wang, Jun-Jie Yang, Tao Zhang, Jing Jing and Yun-Dai Chen*

\begin{abstract}
Background: Epicardial adipose tissue (EAT) is a newly discovered independent risk factor for coronary atherosclerosis. There is a scarcity of information on the reduction of EAT volume to reduce atherosclerosis risk. Coronary computed tomography angiography (CCTA) has emerged as a noninvasive imaging method for the analysis of coronary atherosclerosis and EAT volume. The purpose of this trial is to determine whether olmesartan medoxomil is effective at both treatment of coronary atherosclerosis progression and EAT volume reduction in patients with coronary atherosclerosis detected by CCTA.

Methods/design: This study is a prospective, single-center, open-label, randomized controlled clinical trial aimed at exploring the efficacy of olmesartan medoxomil on coronary atherosclerosis and EAT. A total of 194 patients with coronary stenosis greater than $30 \%$ and less than $70 \%$ detected by CCTA will be randomly divided into olmesartan medoxomil or conventional antihypertensive medication groups (1:1 ratio). The primary outcome measures include coronary atherosclerosis progression and EAT volume reduction, as detected by CCTA at 12 months. The secondary outcome measures include the levels of blood lipids, glucose, high-sensitivity C-reactive protein, IL-6, monocyte chemotactic protein 1, TNF-a, matrix metalloproteinase 9, NO, endothelin 1, adiponectin, and leptin at baseline and after 6 and 12 months.

Discussion: Treatments aimed at reducing EAT volume can eventually achieve an antiatherosclerotic effect. This is the first trial designed to explore the effect of olmesartan medoxomil on both coronary atherosclerosis progression and EAT volume reduction in patients with coronary atherosclerosis detected by CCTA.
\end{abstract}

Trial registration: ClinicalTrials.gov: NCT02360956.

Keywords: Coronary atherosclerosis, Coronary computed tomography angiography, Epicardial adipose tissue

\footnotetext{
* Correspondence: cyundai301@126.com

Department of Cardiology, Chinese PLA General Hospital, Beijing 100853,

China
} 


\section{Background}

Epicardial adipose tissue and coronary atherosclerosis Epicardial adipose tissue (EAT) is directly deposited around the pericardium and coronary artery. By autocrine and paracrine means, EAT can generate various kinds of cytokines, inflammatory mediators and free fatty acids. These biological indicators can affect the state of coronary endothelial function and promote inflammation and oxidative stress, which finally aggravate the progression of coronary atherosclerosis [1-3]. A significant amount of clinical research has been conducted to investigate the link between EAT and coronary atherosclerosis. It was claimed that calcified plaque progression in patients without coronary artery disease and in patients with type 2 diabetes mellitus were associated with a larger EAT volume $[4,5]$. Epicardial adipose tissue is also an independent predictor of significant coronary stenosis and is independently associated with high-risk coronary plaque features, such as low CT attenuation plaque, thin-cap fibroatheroma and positive remodeling [6]. Not only is EAT, as a special visceral fat, correlated with the increased development of coronary artery atherosclerosis, but it is also associated with adverse coronary events [7].

\section{Treatment of epicardial adipose tissue and coronary atherosclerosis}

There are ample studies exploring the progression of coronary atherosclerosis following pharmacological manipulation. At the time of writing, the use of statins is recognized as an effective treatment; statins can result in decreases in plaque and necrotic core volume, and can also significantly reduce the progression of low attenuation plaque (<30 Hounsfield units) and non-calcified plaque $[8,9]$. Other drugs, such as dipeptidyl-peptidase 4 inhibitors [10], a PPAR $\gamma$ agonist (pioglitazone) [11], atorvastatin plus ezetimibe [12], olmesartan [13], have also been reported to have antiatherosclerotic effects, although the effects have not been verified by large-scale studies. As studies show that EAT volume is associated with plaque progression and cardiovascular adverse events, treatments aimed at reducing EAT volume may finally achieve an antiatherosclerotic, preventive effect. However, at the time of writing, limited studies have aimed at reducing both EAT and plaque volume to achieve an antiatherosclerotic effect. A serial coronary computed tomography angiography (CCTA) study recently indicated that intensive statin therapy can reduce the EAT volume of Europeans, but the study failed to demonstrate a relationship among EAT volume reduction, coronary atherosclerosis progression and clinical prognosis [14]; moreover, intensive statin therapy might not be appropriate for Asians. The epidemiological studies and clinical researches show that Asians may have poorer tolerability and safety to intensive statins than white people, owing to genetic differences (variants in structure or polymorphisms) in pharmacokinetics and pharmacodynamics properties [15-17]. It has been claimed that polymorphic variants in cytochrome P450 (CYP450) families that were associated with statin metabolism might result in varying rates of metabolic clearance. CYP450 2C19 slow metabolizer phenotype was reported to be present in approximately $16 \%$ of Asians compared with only about $3 \%$ of white people [18]. Polymorphic variants in the predominant CYP450 isoform, CYP450 3A4, were reported to be associated with a functional decrease in the enzyme's activity in dyslipidemic Chinese patients [19]. The HPS2THRIVE study recently also indicated that, using samedose statin treatment, an excess of increased alanine aminotransferase was seen mainly among Chinese patients (more than three consecutive values above the upper limit of normal of $0.24 \%$ /year compared with $0.02 \% /$ year in Europe) [20]. Moreover, the morbidity of chronic hepatitis B was high in china with nearly 90 million infections. For these reasons, intensive statin therapy may result in higher hepatotoxicity in Asian populations than in white populations, so low- to moderate- dose statin therapy might be more appropriate for Asian populations [15, 16]. Subjects who undergo weight loss exercise, bariatric surgery, or low-dose aspirin therapy can also reduce EAT volume or inflammation, but the effects are weak and these treatments cannot achieve good results in patients with coronary atherosclerosis progression [21-24]. Our aim is to find a drug that reduces EAT volume while inhibiting the progression of coronary atherosclerosis.

In recent years, studies have confirmed that olmesartan medoxomil can improve endothelial function, resist thrombosis, improve tissue reconstruction, and resist oxidative stress to achieve atherosclerosis resistance [13, 25-28]. The latest research shows that olmesartan medoxomil can better inhibit rat epididymal adipose cell hypertrophy and inflammatory reactions [29]. Therefore, we hypothesized that olmesartan medoxomil may also reduce EAT volume, finally achieving an anti-atherosclerosis effect.

\section{EAT and coronary atherosclerosis imaging with computed tomography}

Compared with such invasive methods as intravascular ultrasound, virtual histology intravascular ultrasound, optical coherence tomography, and fractional flow reserve, CCTA has emerged as a noninvasive imaging method that analyzes both coronary atherosclerosis and EAT volume $[14,30]$. To date, ample CCTA studies have explored the progression of coronary atherosclerosis following pharmacological manipulation. Thus, in this study, using CCTA as a noninvasive method to analyze both coronary atherosclerosis progression and EAT volume is of significant clinical value. 


\section{Aims of the main study}

The purpose of this study is to determine whether olmesartan medoxomil is effective on both the treatment of coronary atherosclerosis progression and EAT volume reduction in patients with coronary atherosclerosis detected by CCTA.

\section{Aims of the anti-atherosclerosis mechanism study}

1. To explore the relationship between coronary atherosclerosis progression and EAT volume reduction.

2. To explore the effect of olmesartan medoxomil on serum levels of blood lipids, glucose, circulating surrogate markers of atherosclerosis inflammation, including high-sensitivity C-reactive protein, IL-6, monocyte chemotactic protein 1 (MCP-1), TNF- $\alpha$, and matrix metalloproteinase 9 (MMP-9), circulating surrogate markers of endothelial function, including NO and endothelin 1 (ET-1), and circulating surrogate markers of adipose tissue inflammation and metabolism, including adiponectin and leptin at baseline and after 6 and 12 months.

\section{Methods/design}

\section{Study design}

This study is a prospective, single-center (Chinese PLA General Hospital, Beijing, China), open-label, randomized controlled trial of the efficacy of olmesartan medoxomil on coronary atherosclerosis and EAT. Consecutive patients with coronary stenosis greater than $30 \%$ and less than $70 \%$ detected by CCTA will be randomly assigned to olmesartan medoxomil or conventional antihypertensive medication groups (1:1 ratio). Coronary computed tomography angiography will be conducted at the Department of Cardiology (Chinese PLA General Hospital). Primary outcome measures include coronary atherosclerosis progression and EAT volume reduction, as detected by CCTA, at 12 months. Secondary outcome measures include levels of blood lipids, glucose, high-sensitivity Creactive protein, IL-6, MCP-1, TNF- $\alpha$, MMP-9, NO, ET-1, adiponectin, and leptin at baseline and after 6 and 12 months. The study design is summarized in Fig. 1.

\section{Clinical inclusion and exclusion criteria}

Clinical inclusion criteria are:

- Age between 18 and 75 years;

- Coronary artery stenosis between $30 \%$ and $70 \%$ determined by CCTA in essential hypertension patients;

- Resting diastolic blood pressure between 90 and $110 \mathrm{mmHg}$

- Type A and B for coronary artery vascular lesions.
Clinical exclusion criteria are:

- Secondary hypertension;

- Coronary artery stenosis less than $30 \%$ or greater than $70 \%$, as determined by CCTA;

- Severe arrhythmia;

- Severe cardiac insufficiency or left ventricular dysfunction (left ventricular ejection fraction $<30 \%$ );

- Severe hepatic or kidney insufficiency;

- Resting systolic blood pressure $>200 \mathrm{mmHg}$ or resting diastolic blood pressure $>110 \mathrm{mmHg}$;

- Contraindications to treatment with olmesartan medoxomil (allergy, glaucoma, digestive ulcer, currently taking phosphodiesterase-5 inhibitor);

- Severe calcification, distortion or type $\mathrm{C}$ coronary artery vascular lesions;

- Pregnancy;

- Unwillingness or inability to provide informed consent.

\section{Randomization}

Information regarding the study will be provided to the patient at the Department of Cardiology. Once informed consent is obtained, the patient will be randomized at the Department of Cardiology. Subjects will be randomized to either olmesartan medoxomil or conventional antihypertensive medication groups (1:1 ratio). Participants will be randomized before the first treatment using a blocked randomization procedure (computerized random numbers) and will incorporate minimization to ensure matching for age, sex, body mass index, and hypertension grade.

\section{Ethical considerations}

The Chinese PLA General Hospital Ethics Committee approved this study on 12 December 2014 (reference number S2014-119-01). This study complies with the Declaration of Helsinki. Informed consent will be obtained from all participating patients. Upon signing informed consent, patients' data will be populated as per protocol.

\section{Details of CCTA examination CCTA examination procedure}

CCTA will be performed on a dual-source CT scanner (Somatom Definition Flash, Siemens Healthcare, Forchheim, Germany). All enrolled patients will be instructed in the breath-holding technique before CCTA to minimize breathing artifacts. Three minutes before CCTA, all patients will be given $0.5 \mathrm{mg}$ nitroglycerin sublingually to dilate the coronary artery. The scan range is from the carina or the pulmonary artery segment down to $1 \mathrm{~cm}$ below the diaphragm. Electrocardiography is continuously performed throughout the entire examination for each 


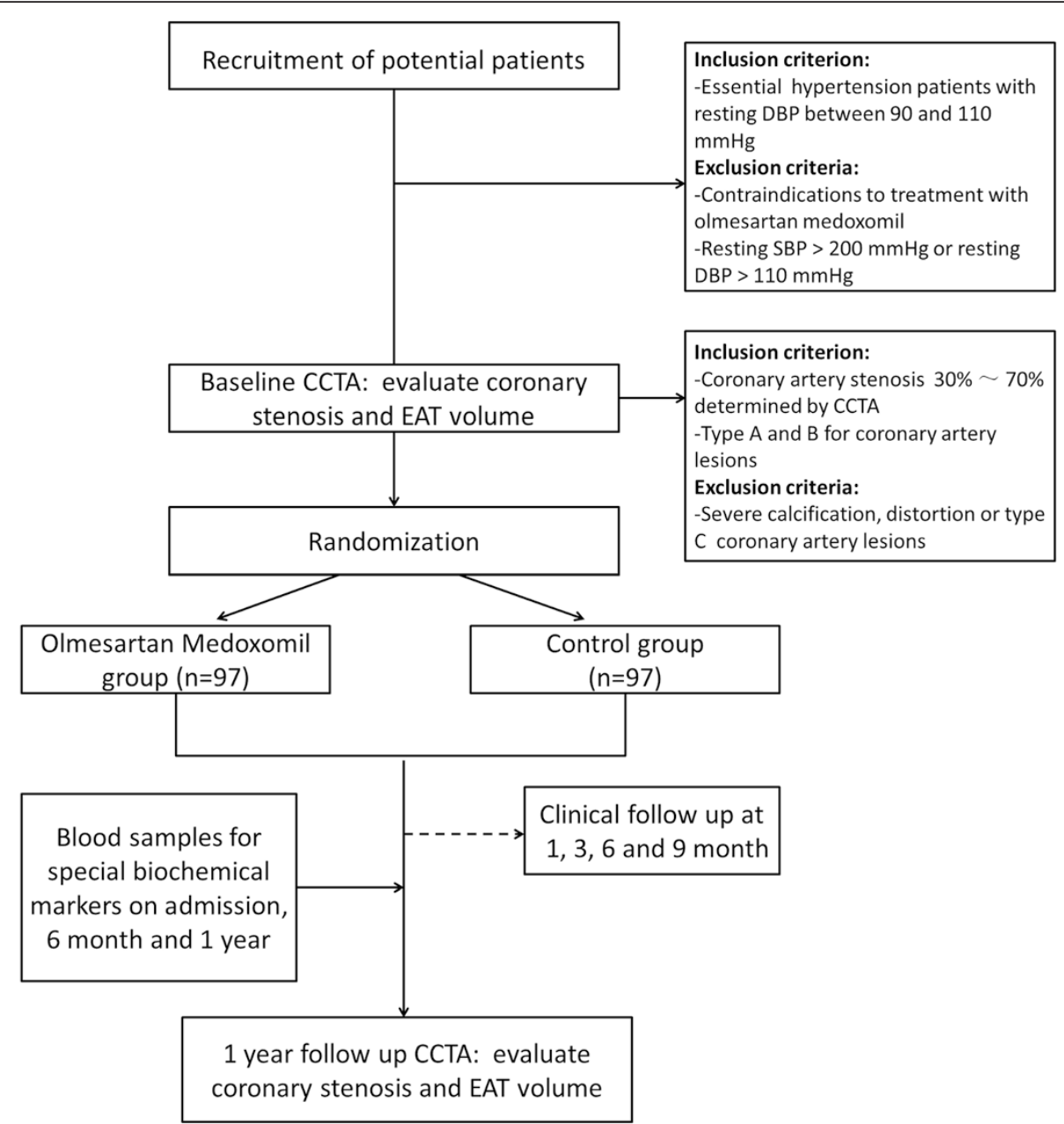

Fig. 1 Study flowchart. CCTA, coronary computed tomography angiography; DBP, diastolic blood pressure; EAT, epicardial adipose tissue; SBP, systolic blood pressure

patient. A dual-head power injector (SCT 210, Medrad, USA) and a nonionic contrast medium (Ultravist ${ }^{\circ}, 370 \mathrm{mg}$ I /ml, Schering AG, Guangzhou, China) will be used. The collimation is $2 \times 128 \times 0.6 \mathrm{~mm}$, the gantry rotation time is $0.28 \mathrm{~ms}$, the slice thickness is $0.6 \mathrm{~mm}$, the tube voltage is 80 to $120 \mathrm{kV}$ (modified using a care kV, Siemens Medical Solutions, Forchheim, Germany), and the tube current is 290 to $560 \mathrm{mAs} /$ rotation (scout-based automatic reference tube current selection - CareDose 4D, Siemens Medical Solutions, Forchheim, Germany). For double flash acquisition, the pitch is 3.4, and for retrospectively ECGtriggered spiral acquisition, the pitch will vary depending on the patient's heart rate. Total estimated radiation dose for the patient will be recorded.

Patients with a heart rate $\leq 70$ beats/min will be evaluated using double prospective ECG-gated high-pitch CT angiography (double flash mode). For the double flash protocol, the contrast-enhanced CCTA protocol is as follows: a test bolus scan will be performed at the level of the aortic root with administration of $15 \mathrm{ml}$ of contrast medium into the right antecubital vein at a rate of $5.0 \mathrm{ml} / \mathrm{s}$, followed by an injection of $20 \mathrm{ml}$ of saline flush at the same flow rate to obtain a peak enhancement time curve. The double flash acquisition triggered scan time is $4 \mathrm{~s}$ after the peak enhancement time. After calculating the triggered scan time, double flash acquisition will be performed by injecting 60 to $90 \mathrm{ml}$ contrast medium at a rate of $5.0 \mathrm{ml} / \mathrm{s}$, immediately followed by $35 \mathrm{ml}$ of 70/30 contrast/saline material mixture and $50 \mathrm{ml}$ saline bolus at the same flow rate. The first scan will begin at $60 \%$ of the R-R interval from the craniocaudal direction. The second scan will be acquired at $30 \%$ of the R-R interval $3 \mathrm{~s}$ after the first scan during the same contrast injection time.

Patients with a heart rate $>70$ beats/min will be evaluated using retrospectively ECG-triggered spiral acquisition. The retrospective protocol is as follows: 60 to $90 \mathrm{ml}$ contrast medium is injected into the antecubital vein at a rate of $5.0 \mathrm{ml} / \mathrm{s}$, immediately followed by $50 \mathrm{ml}$ saline solution at the same flow rate. Bolus tracking is 
used, and the region of interest is set at the root of the ascending aorta. We will perform the scan with a delay of $5 \mathrm{~s}$ after the root of the ascending aorta reaches a threshold of 100 Hounsfield units. For this scan mode, the acquisition is from $30 \%$ to $80 \%$ of the R-R interval.

\section{CCTA image post-processing}

All CCTA data will be sent to the Syngo Multi-Modality Workplace for post-processing. Two independent experienced observers who are unaware of the patients' clinical information will evaluate the CCTA data in different modes, including maximum intensity projection, volume rendering, curved-planar reconstructions, and the original transaxial images. Disagreements in data analysis between the two readers will be resolved by consensus reading.

\section{Epicardial adipose tissue quantification}

The EAT volume will be measured by two experienced radiologists using the same sets of images acquired for the CCTA. The radiologists will be blinded to the purpose of the study, clinical characteristics and patients' anthropometric data.

The EAT volume is defined as the total amount of adipose tissue deposited between the surface of the heart and the visceral pericardium. The region of interest in measuring the EAT volume includes the heart and the surrounding EAT. By manually tracing the epicardium contours in the axial slices from the bifurcation of the pulmonary artery to the diaphragm, the EAT volume is analyzed. The pericardium contour is traced every $10 \mathrm{~mm}$, from the lower visible level of the pulmonary artery bifurcation until the top level of the pulmonary valve, for every $20 \mathrm{~mm}$ until the first slice where the diaphragm becomes visible, and for every $10 \mathrm{~mm}$ from this point until the last slice where the pericardium is still visible [31]. The pericardium contour is manually outlined by the radiologists, and then the software (Syngo Volume, Siemens Medical Solutions) automatically calculates the total EAT volume. Computed tomography attenuation ranging from -195 to 45 Hounsfield units is applied to isolate the EAT from other tissues. Mediastinal adipose tissue and pericardial adipose fat (fat deposit outside the visceral pericardium and on the external surface of the parietal pericardium) are excluded from the analysis. For the assessment of interobserver agreement, we will randomly select 50 patients, and all EAT measures will be assessed by two experienced radiologists blinded to the other radiologist's measurements.

Definition of coronary atherosclerosis plaque progression We will use QAngio CT post-processing software (QAngioCT Research Edition version 2.1.0, Medis Medical
Imaging Systems, Leiden, the Netherlands) to evaluate all CCTA data. All three vessels will be assessed in each patient using the 15-segment American Heart Association model for coronary segment classification [32]. Only segments with a diameter $\geq 2.0 \mathrm{~mm}$ and without stent implantation will be considered for analysis. Parameters including minimal lumen diameter, percent diameter stenosis, minimum lumen area, plaque burden, plaque volume, vascular remodeling index, plaque type classification (calcification, necrosis, fiber, and fiber lipid plaques) based on segments will be analyzed using QAngio CT postprocessing software.

Coronary atherosclerosis progression is defined as $\geq 10 \%$ diameter reduction or progression of a pre-existing coronary stenosis or $\geq 0.2 \mathrm{~mm}$ reduction or progression of the minimal luminal diameter in the lesion [30].

\section{Medication intervention and control protocols}

All patients should accept lifestyle interventions and conventional anti-atherosclerosis treatment. The low-density lipoprotein cholesterol level should be controlled below $100 \mathrm{mg} / \mathrm{dl}$, and the blood pressure should be controlled below 140/90 $\mathrm{mmHg}$.

In the olmesartan medoxomil group, the usual recommended starting dose of olmesartan medoxomil is $20 \mathrm{mg}$ once daily when used as monotherapy in patients who are not volume-contracted. For patients requiring further reduction in blood pressure after 2 weeks of therapy, the dose of olmesartan medoxomil may be increased to $40 \mathrm{mg}$. Doses above $40 \mathrm{mg}$ do not appear to have a greater effect. Twice-daily dosing offers no advantage over the same total dose given once daily.

In the control group, any antihypertensive medication alone or in combination, including calcium channel blockers, diuretics, beta blockers, or other antihypertensive medication except angiotensin-converting enzyme inhibitors or angiotensin II receptor blockers can be used. The drug dose must be individualized. The patients should take the antihypertensive drugs according to the doctors' recommendations.

\section{Study outcomes}

The primary outcome measures are coronary atherosclerosis progression and EAT volume changes, as detected by CCTA at 12 months.

The secondary outcome measures include

1. The relationship between coronary atherosclerosis and EAT;

2. Serum levels of blood lipids, glucose, circulating surrogate markers of atherosclerotic inflammation including high-sensitivity C-reactive protein, IL-6, MCP-1, TNF- $\alpha$, and MMP-9, individual circulating 
surrogate markers of endothelial function including NO and ET-1, and individual circulating surrogate markers of adipose tissue inflammation and metabolism including adiponectin and leptin at baseline and after 6 and 12 months.

\section{Biomarkers}

Two 10-ml samples of blood will be collected from the antecubital vein by a trained nurse for each individual. Fasting blood samples will be obtained between 7:00 a.m. and 12:00 noon to control for possible diurnal variations. Blood samples will be centrifuged at $4{ }^{\circ} \mathrm{C}$ and 3,000 rpm for $15 \mathrm{~min}$. The serum will be sampled and stored at $-80{ }^{\circ} \mathrm{C}$ until analysis. Traditional cardiovascular blood risk markers, including fasting blood glucose, triglycerides, total cholesterol, and high- and low-density lipoprotein cholesterol will be assessed. The following proinflammatory markers will be assessed: high-sensitivity C-reactive protein, IL-6, TNF- $\alpha$, MCP-1, and MMP-9. In addition, NO and ET-1 will be measured, to assess endothelial function. Markers of adipose tissue inflammation and metabolism, including adiponectin and leptin, will also be assessed. These markers will be measured at baseline and 6 and 12 months after treatment.

\section{Follow-up}

Clinical follow-up will take place at 1 month ( \pm 1 week), 3 months ( \pm 2 weeks), 6 months ( \pm 2 weeks), 9 months ( \pm 30 days), and 1 year ( \pm 30 days) by clinical visit or phone interview.

At baseline and 6-month ( \pm 2 weeks) and 1 year ( \pm 30 days) follow-ups, all patients will provide venous blood for detection of blood lipids, glucose, high-sensitivity C-reactive protein, IL-6, MCP-1, TNF- $\alpha$, NO, ET-1, MMP9 , adiponectin, and leptin.

At 1 year ( \pm 30 days) follow-up, all patients will undergo CCTA (with QAngio CT post-processing software).We anticipated a patient drop-out rate of $10 \%$.

\section{Sample size calculation}

This trial is an open-label randomized clinical trial, so patients will randomly be assigned to olmesartan medoxomil or conventional antihypertensive medication groups (1:1 ratio). The purpose of this study is to verify that olmesartan medoxomil is effective in the treatment of coronary atherosclerosis progression and EAT volume reduction in patients with coronary atherosclerosis detected by CCTA. We also want to elucidate the relationship between coronary atherosclerosis and EAT. The mechanism by which olmesartan medoxomil inhibits coronary atherosclerosis progression will be studied by detecting the serum levels of blood lipids, glucose, circulating surrogate markers of atherosclerosis inflammation including highsensitivity C-reactive protein, IL-6, MCP-1, TNF- $\alpha$, and
MMP-9, circulating surrogate markers of endothelial function, including NO and ET-1, and circulating surrogate markers of adipose tissue inflammation and metabolism, including adiponectin and leptin.

Studies on coronary atherosclerosis progression rate have had differing results. The combined results of multiple studies indicate that the conventional mean coronary atherosclerosis progression rate is about $30 \%$ [33-35]. We hypothesize that additional olmesartan medoxomil use will reduce the coronary atherosclerosis progression rate to $13 \%$ [36-40]. Using double-side inspection, $\alpha=0.05, \beta=0.2$, we calculate a total sample size of 176 cases; considering the expected loss to follow-up to be $10 \%$, the number of cases to be included should be at least $176 \times(1+10 \%)=194$. Therefore, we aim for 97 cases of each group.

\section{Statistical analysis}

Continuous variables will be described using means and standard deviations or median and range in case of asymmetric distribution of data. Categorical variables will be presented using frequency distribution. Univariate analyses will be conducted using chi-square and $t$ tests for independent samples. A multiple logistic regression analysis will be performed to correlate coronary atherosclerosis progression with clinical variables and EAT volume, including treatment groups. Statistical significance will be considered for $P<$ 0.05. A statistical package (SPSS 16.0) will be used for analysis. The individual will be considered the unit of analysis.

\section{Discussion}

To date, there are ample CCTA studies exploring the progression of coronary atherosclerosis following pharmacological manipulation. As studies show that EAT volume is associated with plaque progression and cardiovascular adverse events, treatments aimed at reducing EAT volume may finally achieve an antiatherosclerotic, preventive effect. However, at the time of writing, only a limited number of studies have aimed to reduce both EAT and plaque volume to achieve a preventive effect against atherosclerosis. The novelty of this study is that we intend to explore the effect of olmesartan medoxomil on both plaque volume and epicardial fat. This study will accomplish two goals: (1) it will explain the relationship between EAT volume and coronary atherosclerosis progression and (2) it will verify the effect of olmesartan medoxomil on EAT volume reduction and coronary atherosclerosis progression.

If these hypotheses are supported, the study findings will have significant implications related to clinical practice. Evidence that olmesartan medoxomil is effective on EAT volume reduction and coronary atherosclerosis progression would be very attractive to clinicians and patients. This may further contribute to the care of patients with coronary heart disease. 


\section{Trial status}

\section{Recruitment for the study is currently ongoing. Patient recruitment began in December 2014.}

\section{Abbreviations}

CCTA: coronary computed tomography angiography; CT: computed tomography; CYP450: cytochrome P450; EAT: epicardial adipose tissue; ET-1: endothelin 1; IL-6: interleukin 6; MCP-1: monocyte chemotactic protein 1; MMP-9: matrix metalloproteinase 9; TNF-a: tumor necrosis factor a.

\section{Competing interests}

The authors declare no competing interests.

\section{Authors' contributions}

YZ conceived the study and drafted this manuscript, and will also conduct the CCTA examinations and image analysis. FT and JJ will conduct patient recruitment. JJY will conduct the CCTA examinations and image analysis. JW and TZ performed the statistical analysis and helped to draft this manuscript. YDC is the Head of the Department of Cardiology, conceived the study, and drafted this manuscript. All authors read and approved the final manuscript.

\section{Acknowledgements}

We are grateful to numerous colleagues who are providing clinical and research support in treating and following the patients included in this trial. This work was confirmed as Scientific Innovation Research supported by Chinese PLA General Hospital. The funding body had no role in designing the study, nor will it be involved in the collection, analysis, or interpretation of data.

Received: 12 May 2015 Accepted: 1 December 2015

Published online: 06 January 2016

\section{References}

1. lacobellis G, Corradi D, Sharma AM. Epicardial adipose tissue: anatomic, biomolecular and clinical relationships with the heart. Nat Clin Pract Cardiovasc Med. 2005;2(10):536-43.

2. lacobellis G, Bianco AC. Epicardial adipose tissue: emerging physiological, pathophysiological and clinical features. Trends Endocrinol Metab. 2011;22(11):450-7

3. Sacks HS, Fain JN. Human epicardial adipose tissue: a review. Am Heart J. 2007;153(6):907-17.

4. Rosito GA, Massaro JM, Hoffmann U, Ruberg FL, Mahabadi AA, Vasan RS, et al. Pericardial fat, visceral abdominal fat, cardiovascular disease risk factors, and vascular calcification in a community-based sample: the Framingham Heart Study. Circulation. 2008;117(5):605-13.

5. Yerramasu A, Dey D, Venuraju S, Anand DV, Atwal S, Corder R, et al. Increased volume of epicardial fat is an independent risk factor for accelerated progression of sub-clinical coronary atherosclerosis. Atherosclerosis. 2012;220(1):223-30

6. Rajani $R$, Shmilovich $H$, Nakazato $R$, Nakanishi $R$, Otaki $Y$, Cheng VY, et al. Relationship of epicardial fat volume to coronary plaque, severe coronary stenosis, and high-risk coronary plaque features assessed by coronary CT angiography. J Cardiovasc Comput Tomogr. 2013;7(2):125-32

7. Mahabadi AA, Berg MH, Lehmann N, Kalsch H, Bauer M, Kara K, et al. Association of epicardial fat with cardiovascular risk factors and incident myocardial infarction in the general population: the Heinz Nixdorf Recall Study. J Am Coll Cardiol. 2013;61(13):1388-95.

8. Inoue K, Motoyama S, Sarai M, Sato T, Harigaya H, Hara T, et al. Serial coronary CT angiography-verified changes in plaque characteristics as an end point: evaluation of effect of statin intervention. JACC CardiovasC Imaging. 2010;3(7):691-8.

9. Zeb I, Li D, Nasir K, Malpeso J, Batool A, Flores F. Effect of statin treatment on coronary plaque progression - a serial coronary CT angiography study. Atherosclerosis. 2013;231(2):198-204.

10. Shah Z, Kampfrath T, Deiuliis JA, Zhong J, Pineda C, Ying Z, et al. Long-term dipeptidyl-peptidase 4 inhibition reduces atherosclerosis and inflammation via effects on monocyte recruitment and chemotaxis. Circulation. 2011;124(21):2338-49.
11. Mani P, Uno K, St John J, Kupfer S, Perez A, Tuzcu EM, et al. Favorable impact on LDL particle size in response to treatment with pioglitazone is associated with less progression of coronary atherosclerosis in patients with type 2 diabetes. J Am Coll Cardiol. 2015;66(3):328-9.

12. Tsujita K, Sugiyama S, Sumida H, Shimomura H, Yamashita T, Yamanaga K, et al. Impact of dual lipid-lowering strategy with ezetimibe and atorvastatin on coronary plaque regression in patients with percutaneous coronary intervention: the multicenter randomized controlled PRECISE-IVUS trial. J Am Coll Cardiol. 2015;66(5):495-507.

13. Hirohata A, Yamamoto K, Miyoshi T, Hatanaka K, Hirohata S, Yamawaki $\mathrm{H}$, et al. Impact of olmesartan on progression of coronary atherosclerosis a serial volumetric intravascular ultrasound analysis from the OLIVUS (impact of OLmesarten on progression of coronary atherosclerosis: evaluation by intravascular ultrasound) trial. J Am Coll Cardiol. 2010:55(10):976-82.

14. Alexopoulos N, Melek BH, Arepalli CD, Hartlage GR, Chen Z, Kim S, et al. Effect of intensive versus moderate lipid-lowering therapy on epicardial adipose tissue in hyperlipidemic post-menopausal women: a substudy of the BELLES trial (beyond endorsed lipid lowering with EBT scanning). J Am Coll Cardiol. 2013;61(19):1956-61.

15. Expert Panel of 2014 China Cholesterol Education Program Experts Recommendations for Management of Dyslipidemia, Working Group on Blood Lipid and Atherosclerosis of Editorial Board of Chinese Journal of Cardiology, Epidemiology Group of Chinese Society of Cardiology, Expert Panel of 2014 China Cholesterol Education Program Experts Recommendations for Management of Dyslipidemia, Working Group on Blood Lipid and Atherosclerosis of Editorial Board of Chinese Journal of Cardiology, Epidemiology Group of Chinese Society of Cardiology. 2014 China cholesterol education program experts recommendations on management of dyslipidemia. Zhonghua Xin Xue Guan Bing Za Zhi. 2014; 42(8):633-6.

16. Stone NJ, Robinson JG, Lichtenstein AH, Bairey Merz CN, Blum CB, Eckel RH, et al. 2013 ACC/AHA guideline on the treatment of blood cholesterol to reduce atherosclerotic cardiovascular risk in adults: a report of the American College of Cardiology/American Heart Association Task Force on Practice Guidelines. J Am Coll Cardiol. 2014;63(25 Pt B):2889-934.

17. Liao JK. Safety and efficacy of statins in Asians. Am J Cardiol. 2007;99(3):410-4.

18. Poolsup N, Li Wan Po A, Knight TL. Pharmacogenetics and psychopharmacotherapy. J Clin Pharm Ther. 2000;25(3):197-220.

19. Wang A, Yu BN, Luo CH, Tan ZR, Zhou G, Wang LS, et al. Ile118Val genetic polymorphism of CYP3A4 and its effects on lipid-lowering efficacy of simvastatin in Chinese hyperlipidemic patients. Eur J Clin Pharmacol. 2005;60(12):843-8.

20. HPS2-THRIVE. Randomized placebo-controlled trial in 25673 high-risk patients of ER niacin/laropiprant: trial design, pre-specified muscle and liver outcomes, and reasons for stopping study treatment. Eur Heart J. 2013;34(17):1279-91

21. Kim MK, Tomita T, Kim MJ, Sasai H, Maeda S, Tanaka K. Aerobic exercise training reduces epicardial fat in obese men. J Appl Physiol (1985). 2009:106(1):5-11.

22. lacobellis G, Singh N, Wharton S, Sharma AM. Substantial changes in epicardial fat thickness after weight loss in severely obese subjects. Obesity (Silver Spring). 2008;16(7):1693-7.

23. Willens HJ, Byers P, Chirinos JA, Labrador E, Hare JM, de Marchena E. Effects of weight loss after bariatric surgery on epicardial fat measured using echocardiography. Am J Cardiol. 2007;99(9):1242-5.

24. Grosso AF, de Oliveira SF, Higuchi Mde L, Favarato D, Dallan LA, da Luz PL. Synergistic anti-inflammatory effect: simvastatin and pioglitazone reduce inflammatory markers of plasma and epicardial adipose tissue of coronary patients with metabolic syndrome. Diabetol Metab Syndr. 2014;6(1):47.

25. Miyoshi T, Hirohata A, Usui S, Yamamoto K, Murakami T, Komatsubara I, et al. Olmesartan reduces inflammatory biomarkers in patients with stable coronary artery disease undergoing percutaneous coronary intervention: results from the OLIVUS trial. Heart Vessels. 2014;29(2):178-85.

26. Mason RP. Optimal therapeutic strategy for treating patients with hypertension and atherosclerosis: focus on olmesartan medoxomil. Vasc Health Risk Manag. 2011;7:405-16.

27. Ferrario C. Effect of angiotensin receptor blockade on endothelial function: focus on olmesartan medoxomil. Vasc Health Risk Manag. 2009:5(1):301-14.

28. Fliser D, Buchholz K, Haller H. Antiinflammatory effects of angiotensin II subtype 1 receptor blockade in hypertensive patients with microinflammation Circulation. 2004:110(9):1103-7. 
29. Maeda A, Tamura K, Wakui H, Ohsawa M, Azushima K, Uneda K, et al. Effects of the angiotensin receptor blocker olmesartan on adipocyte hypertrophy and function in mice with metabolic disorders. BioMed Res Int. 2014;2014:946492

30. Boogers MJ, Schuijf JD, Kitslaar PH, van Werkhoven JM, de Graaf FR, Boersma $E$, et al. Automated quantification of stenosis severity on 64-slice CT: a comparison with quantitative coronary angiography. JACC CardiovasC Imaging. 2010;3(7):699-709.

31. Bettencourt N, Toschke AM, Leite D, Rocha J, Carvalho M, Sampaio F, et al. Epicardial adipose tissue is an independent predictor of coronary atherosclerotic burden. Int J Cardiol. 2012;158(1):26-32.

32. Austen WG, Edwards JE, Frye RL, Gensini GG, Gott VL, Griffith LS, et al. A reporting system on patients evaluated for coronary artery disease. Report of the Ad Hoc Committee for Grading of Coronary Artery Disease, Council on Cardiovascular Surgery, American Heart Association. Circulation. 1975:51(4 Suppl):5-40.

33. Zheng JL, Lu L, Hu J, Zhang RY, Zhang Q, Chen QJ, et al. Increased serum YKL-40 and C-reactive protein levels are associated with angiographic lesion progression in patients with coronary artery disease. Atherosclerosis. 2010;210(2):590-5.

34. Han Y, Jing J, Tu S, Tian F, Xue H, Chen W, et al. ST elevation acute myocardial infarction accelerates non-culprit coronary lesion atherosclerosis. Int J Cardiovasc Imaging. 2014;30(2):253-61.

35. Bourantas CV, Garcia-Garcia HM, Farooq V, Maehara A, Xu K, Genereux P, et al. Clinical and angiographic characteristics of patients likely to have vulnerable plaques: analysis from the PROSPECT study. JACC Cardiovasc Imaging. 2013; 6(12):1263-72.

36. Puri R, Nissen SE, Shao M, Ballantyne CM, Barter PJ, Chapman MJ, et al. Coronary atheroma volume and cardiovascular events during maximally intensive statin therapy. Eur Heart J. 2013;34(41):3182-90.

37. Puri R, Nissen SE, Libby P, Shao M, Ballantyne CM, Barter PJ, et al. C-reactive protein, but not low-density lipoprotein cholesterol levels, associate with coronary atheroma regression and cardiovascular events after maximally intensive statin therapy. Circulation. 2013;128(22):2395-403.

38. Nicholls SJ, Ballantyne CM, Barter PJ, Chapman MJ, Erbel RM, Libby P, et al. Effect of two intensive statin regimens on progression of coronary disease. N Engl J Med. 2011;365(22):2078-87.

39. Christoph M, Herold J, Berg-Holldack A, Rauwolf T, Ziemssen T, Schmeisser $A$, et al. Effects of the PPARy agonist pioglitazone on coronary atherosclerotic plaque composition and plaque progression in non-diabetic patients: a double-center, randomized controlled VH-IVUS pilot-trial. Heart Vessels. 2015;30(3):286-95.

40. Clementi F, Di Luozzo M, Mango R, Luciani G, Trivisonno A, Pizzuto F, et al Regression and shift in composition of coronary atherosclerotic plaques by pioglitazone: insight from an intravascular ultrasound analysis. J Cardiovasc Med (Hagerstown). 2009;10(3):231-7.

\section{Submit your next manuscript to BioMed Central and we will help you at every step:}

- We accept pre-submission inquiries

- Our selector tool helps you to find the most relevant journal

- We provide round the clock customer support

- Convenient online submission

- Thorough peer review

- Inclusion in PubMed and all major indexing services

- Maximum visibility for your research

Submit your manuscript at www.biomedcentral.com/submit

) Biomed Central 